\title{
FRATURA DA ESCÁPULA: RESULTADOS DO TRATAMENTO CIRÚRGICO EM 15 PACIENTES
}

\author{
SCAPULA FRACTURES: OUTCOMES AFTER SURGICAL TREATMENT IN 75 PATIENTS
}

\begin{abstract}
Vincenzo Giordano', Ney Pecegueiro do Amaral ${ }^{2}$, Marcelo Soares ${ }^{5}$, Alexandre Pallottino ${ }^{3}$, Rodrigo Pires e Albuquerque ${ }^{4}$, José Félix dos Santos $\mathrm{Neto}^{3}$, Felipe Serrão de Souza ${ }^{6}$, Getúlio José Miguel Filho ${ }^{5}$
\end{abstract}

\section{RESUMO}

Objetivo: Apresentar os resultados do tratamento cirúrgico de 15 pacientes com fratura da escápula. Métodos: Avaliação retrospectiva dos resultados clinicos e radiográficos do tratamento cirurgico de fraturas da escápula, em um periodo de dez anos. A avaliação clínica baseou-se tanto no escore de Schofer et al. quanto no Constant-Murley. Na avaliação radiográfica, pesquisouse a existência de perda da redução, pseudartrose ou alterações póstraumáticas. A análise estatística foi puramente descritiva, por meio de cálculo percentual dos achados. Resultados: Todas as fraturas consolidaram em um período médio de 90 dias, conforme documentação radiográfica. Na última consulta ambulatorial, o tempo médio de seguimento foi de 45,6 meses (variando de 14 a 109,2 meses). Nenhum paciente referiu dor constante e incapacitante no ombro operado. O escore médio de Constant- Murley foi de 84 pontos (variando de 76 a 90 pontos). Dos 15 pacientes, $13(86,7 \%)$ retornaram ao nível funcional prévio sem restrição (atividades profissional e recreacionais). Não forma detectados sinais de perda de redução, pseudartrose ou osteo- artrose póstraumática. Não ocorreram penetração ou impacto articular pela presença do material de osteossíntese. Conclusão: Em pacientes com fraturas da escápula, que se enquadrem nos critérios para tratamento cirúrgico, os resultados são bons desde que sejam respeitados os tecidos moles periarticulares e realizado o devido planejamento da via de acesso e do protocolo de reabilitação.

Descritores: Fratura da Escapula; Tratamento Cirurgico; Resultados

\section{ABSTRACT}

Objective: To evaluate the clinical and radiographic outcomes on 15 unstable scapula fractures treated by open reduction and internal fixation. Methods: A retrospective study to evaluate the results of scapula fracture fixation in 15 patients, managed at our Institution during a 10-year period. Shoulder function was recorded in both upper extremities with both the Schofer et al. and the Constant-Murley score. Radiographic analysis was done in terms of loss of reduction, nonunion, and post-traumatic degenerative changes. Statistical analysis was purely descriptive with calculation of percentage. Results: All fractures healed in a mean of 90 days, as documented by simple radiographs. At the last consultation, the mean follow-up was 45.6 months (ranging from 14 to 109,2 months). No patient had constant pain or referred incapacity. The mean Constant-Murley score for the 15 patients was 84 points (ranging from 76 to 90 points). Of the 15 patients, 13 (86.7\%) had returned to their previous employment and recreational activities with-out restrictions. No signs of loss of reduction, nonunion, or post-traumatic degenerative changes were seen. In addition, no ardware impingement or articular penetration was observed in any case. conclusion: The respect to the soft tissue is the cornerstone of proper treatment of this scapular fractures. In our series good results were obtained when appropriate preoperative planning and postoperative rehabilitation has been performed.

Keywords: Scapular Fracture; Surgical Treatment; Outcomes

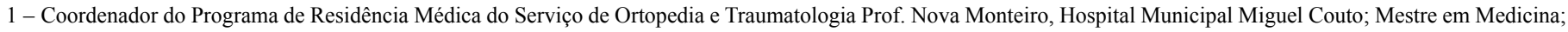
Especialista em Cirurgia do Trauma Ortopédico; Membro Titular da SBOT e da SBTO.

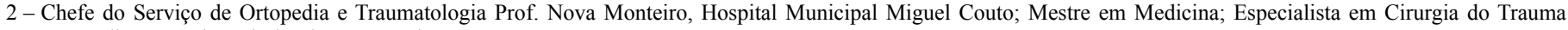
Ortopédico; Membro Titular da SBOT e da SBTO.

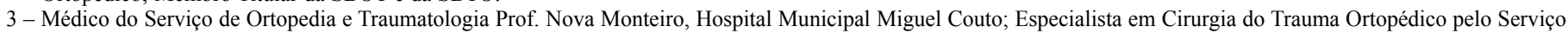
de Ortopedia e Traumatologia Prof. Nova Monteiro, Hospital Municipal Miguel Couto; Membro Titular da SBOT e da SBTO.

4 - Médico do Serviço de Ortopedia e Traumatologia Prof. Nova Monteiro, Hospital Municipal Miguel Couto; Doutor em Medicina; Membro Titular da SBOT.

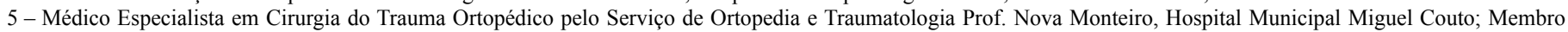
Titular da SBOT e da SBTO.

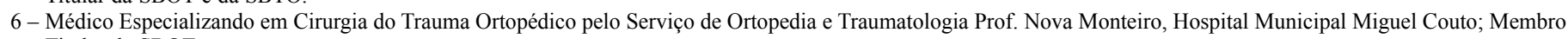
Titular da SBOT.

Trabalho realizado no Serviço de Ortopedia e Traumatologia Prof. Nova Monteiro, Hospital Municipal Miguel Couto, Rio de Janeiro - RJ, Brasil.

Correspondência: Rua Aristides Espínola 11 / 301 - Leblon - 22440-050 - Rio de Janeiro, RJ, Brasil. E-mail: sot.hmmc@terra.com.br

Trabalho recebido para publicação: 15/03/11, aceito para publicação: 15/04/11. 


\section{INTRODUÇÃO}

A ocorrência de fratura da escápula é extremamente incomum, representando aproximadamente de $3 \%$ a $5 \%$ de todas as fraturas da cintura escapular. Acredita-se que os principais motivos da raridade desta lesão sejam o volumoso envelope de partes moles na qual este osso está localizado e a sua mobilidade na caixa torácica $^{(1)}$. Ocorrem predominantemente na população masculina jovem e de meia idade, vítima de trauma de alta energia, que sofrem impacto direto sobre o aspecto póstero-superior e lateral do tronco ${ }^{(1,2)}$. O trauma torácico é a lesão mais comumente associada à fratura da escápula, embora haja um elevado índice de pacientes vítimas de múltiplo trauma ${ }^{(2)}$. Neste cenário, é fundamental que se suspeite de fratura da escápula em todo paciente politraumatizado e com contusão torácica ${ }^{(2)}$.

Uma vez que não se observa com frequência grande desvio entre os fragmentos e a maioria destas lesões ocorre no corpo da escápula, o tratamento geralmente é não cirúrgico ${ }^{(3)}$. A indicação de tratamento conservador em casos instáveis, no entanto, é questionável. Como desvio progressivo pode ser detectado em certos padrões mais instáveis, tem sido recomendado acompanhamento estrito quando se mantém a indicação não cirúrgica nestes casos. Desequilíbrio muscular, fraqueza, rigidez e osteoartrose (OA) póstraumática precoce têm sido relatadas em pacientes que evoluíram com consolidação viciosa após tratamento conservador de padrões instáveis de fratura da escápula.

A primeira descrição de fixação interna de fratura da escápula foi de Lambotte, em 1913. Este autor relatou o tratamento de uma fratura não especificada da espinha da escápula com dois parafusos ${ }^{(4)}$. Depois disto, as indicações de tratamento cirúrgico para as fraturas da escápula foram sendo gradualmente estabelecidas e atualmente as clássicas são: desvio ou translação lateral do corpo maior do que $10,0 \mathrm{~mm}$, angulação do corpo maior do que $40^{\circ}$, desvio do colo da glenoide maior do que $15,0 \mathrm{~mm}$ (com ou sem deformidade angular ou rotacional), desvio articular da glenoide maior do que $5,0 \mathrm{~mm}$ e dupla rotura do complexo suspensório superior do ombro $(\mathrm{CSSO})^{(1,3)}$.

O objetivo dos autores é apresentar os resultados preliminares do tratamento cirúrgico de 15 pacientes com fratura da escápula.

\section{MÉTODOS}

Entre os meses de janeiro de 2000 e janeiro de 2010, foram operados no Serviço de Ortopedia e Traumatologia Prof. Nova Monteiro - Hospital Municipal Miguel Couto 15 pacientes esqueleticamente maduros com fraturas desviadas da escápula. Todos foram admitidos no hospital pelo Setor de Emergência, trazidos pelo sistema médico de resgate do município do Rio de Janeiro, e abordados na fase inicial de como politraumatizados ${ }^{(5)}$. Nas radiografias iniciais, qualquer sinal de trauma torácico, como pneumotórax, hemotórax ou fraturas múltiplas de arco costal, foi pesquisado e, quando necessário, tratado em caráter emergencial. Após a estabilização clínica adequada, a avaliação por imagem foi complementada com outras incidência radiográficas e tomografia computadorizada do tórax e de outras regiões, de acordo com a presença ou a suspeita de lesão associada.

Dos 15 pacientes incluídos neste estudo, 13 eram do sexo masculino e dois do feminino. A média de idade foi de 39 anos, variando de 21 a 57 anos. As fraturas foram classificadas de acordo com o sistema de Ada e Miller ${ }^{(3)}$. Especificamente as fraturas da glenoide foram também classificadas de acordo com Ideberg et $a l .{ }^{(6)}$. Os dados demográficos dos pacientes encontramse na Tabela 1. As Figuras 1 e 2 mostram os padrões de lesão de acordo com as classificações de Ada e Miller e de Ideberg et al., respectivamente ${ }^{(3,6)}$. Um paciente teve rotura do complexo suspensório superior do ombro (CSSO), caracterizada por fratura ipsilateral do terço distal da clavícula e da parte superior da cavidade glenoide.

A indicação do tratamento cirúrgico baseou-se um dos critérios a seguir ${ }^{(7,8)}$ :

(1) fratura do colo da glenoide com desvio superior a $2,0 \mathrm{~cm}$,

(2) fratura do colo da glenoide com angulação superior a $40^{\circ}$,

(3) fratura intra-articular da glenoide com desvio superior a $5,0 \mathrm{~mm}$,

(4) fratura intra-articular da glenoide associada à instabilidade gleno-umeral,

(5) fratura do corpo da escápula com desvio superior a $10,0 \mathrm{~mm}$,

(6) dupla rotura do CSSO. 
Tabela 1 - Dados demográficos dos pacientes.

\begin{tabular}{l|c}
\hline & $\begin{array}{c}\text { Pacientes } \\
\text { (n = 15) }\end{array}$ \\
\hline Idade média (anos) [variação] & $39[21-57]$ \\
Sexo (masculino / feminino) & 13 / 02 \\
Classificação de Ada e Miller & 15 \\
- tipo IIA (colo - vertical) & 03 \\
tipo IIC (colo - horizontal) & 01 \\
- tipo III (glenoide) & 10 \\
- tipo IV (corpo) & 01 \\
Classificação de Ideberg et al. & 10 \\
- tipo 1B (rebordo anterior grande) & 01 \\
- tipo 2 (inferior) & 05 \\
- tipo 3 (superior) & 01 \\
- tipo 5 (horizontal + completa do colo) & 03 \\
\hline
\end{tabular}

Fonte: SOT Prof. Nova Monteiro - HMMC, 2010

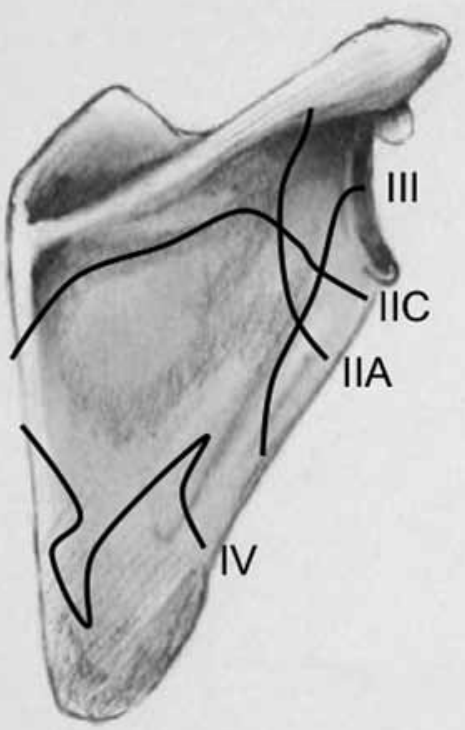

Figura 1 - Classificação de Ada e Miller $(n=15)$.
Os pacientes foram operados entre o terceiro e o sétimo dias de hospitalização, sempre pelo mesmo cirurgião. Em 13 pacientes a fratura foi abordada por via posterior e em dois pela anterior. $\mathrm{O}$ acesso posterior "clássico" de Judet foi utilizado em quatro pacientes e o "modificado" por Obrenskey e Lyman em nove ${ }^{(9,10)}$. $\mathrm{O}$ acesso anterior foi realizado em linha com a prega axilar anterior ${ }^{(11)}$.

Um dos autores conduziu o protocolo de reabilitação no curso pós-operatório. Os pacientes foram rotineiramente vistos em regime ambulatorial com uma, duas, três, seis e 12 semanas e com seis e 12 meses após a alta hospitalar. Apos um ano da cirurgia, os pacientes foram acompanhados anualmente de forma regular.

Na última avaliação ambulatorial, foi solicitado aos pacientes que auto-avaliassem a presença de dor articular no ombro operado e a pontuassem de acordo com os critérios de Schofer et al. ${ }^{(12)}$.

- totalmente sem dor,

- dor ocasional, apenas após grandes esforços,

- dor frequente após grandes esforços,

- dor frequente após esforços leves,

- dor constante.

De forma comparativa, a função dos dois ombros foi avaliada de acordo com o escore de Constant-Murley ${ }^{(13)}$. A análise radiográfica foi realizada em busca de perda a redução, pseudartrose ou OA pós-traumática.

$\mathrm{A}$ análise estatística foi realizada de modo puramente descritivo, por meio do cálculo percentual dos achados.

O Consentimento Informado foi obtido de todos os pacientes no momento da última consulta ambulatorial.
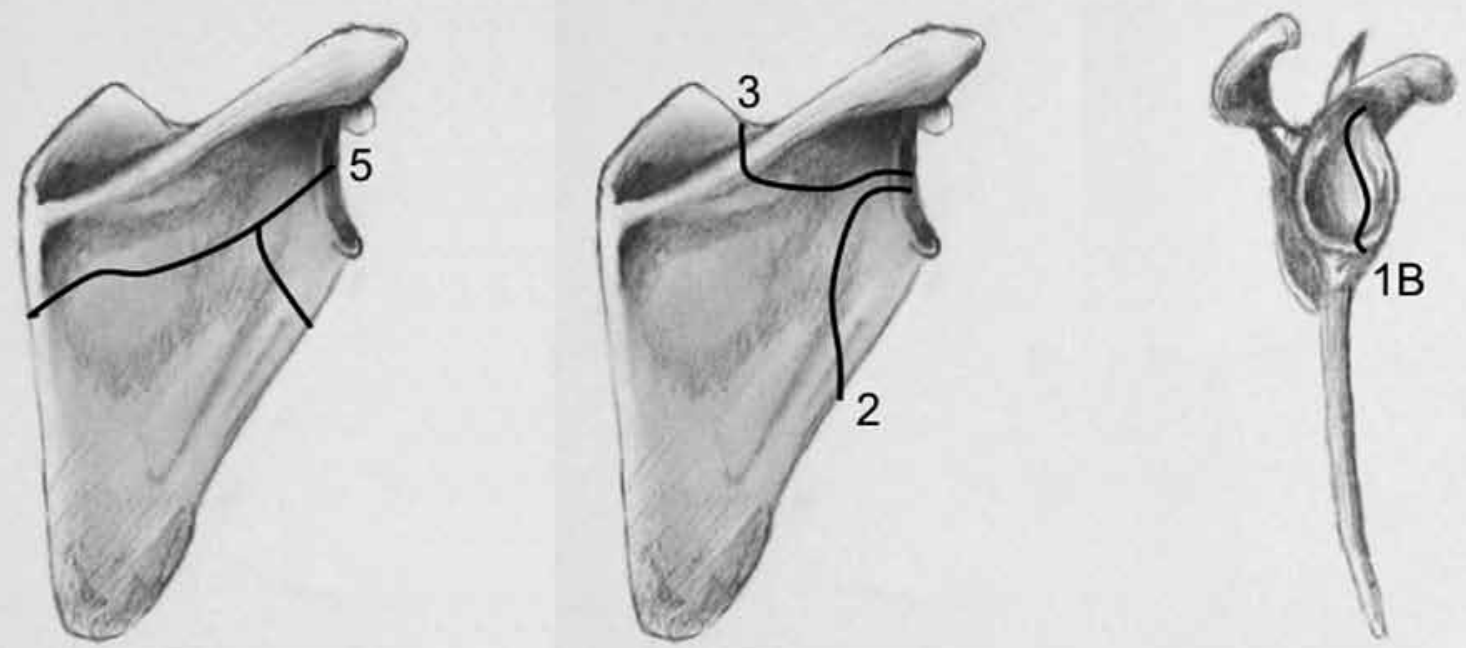

Figura 2 - Classificação de Ideberg et al. $(n=10)$.

Rev Bras Ortop. 2011;46(Suppl 1):28-33 


\section{RESULTADOS}

Todas as fraturas consolidaram em média com 90 dias, conforme documentação radiográfica simples. Uma vez que os pacientes seguiram um protocolo de visitas ambulatoriais pré-determinadas, não foi possível definir precisamente o tempo mínimo e o tempo máximo de consolidação. Na última visita ambulatorial, o tempo médio de seguimento foi de 45,6 meses (variando de 14 a 109,2 meses).

\section{Auto-percepção da dor (critérios de Schofer et al.)}

Dos 15 pacientes, um $(6,7 \%)$ estava totalmente sem dor, $12(80 \%)$ referiam dor ocasional e dois $(13,3 \%)$ tinham dor frequente após grandes esforços. Nenhum paciente queixou-se de dor constante. Não houve necessidade de uso regular de medicação analgésica ou fisioterapia. Nenhum paciente foi afastado por invalidez ou tornou-se dependente do Instituto Nacional de Seguridade Social (INSS).

Consideramos o resultado final quanto à dor satisfatório em $13(86,7 \%)$ pacientes.

\section{Função do ombro (escore de Constant-Murley)}

O escore médio de Constant-Murley foi de 84 pontos (variando de 76 a 90 pontos). Dos 15 pacientes, $13(86,7 \%)$ retornaram ao seu nível funcional prévio, profissional e recreacional, sem restrição. Dois $(13,3 \%)$ pacientes foram incapazes de reassumir sua função profissional ou sua atividade profissional prévias devido a restrições impostas pelo ombro operado.

$\operatorname{Um}(6,7 \%)$ destes pacientes sofreu fratura multifragmentar da escápula (Ada e Miller tipo III / Ideberg et al. tipo 5) e não recuperou a mobilidade articular (elevação frontal e lateral, e rotação externa), apesar de referir dor quando submetido a grandes esforços. Além disto, este paciente evoluiu com dor complexa regional dolorosa do tipo I no antebraço contralateral após ter sofrido fraturas ipsilaterais da diáfise dos ossos do antebraço e do ossos do carpo. Atualmente está em acompanhamento fisioterápico, sem programação de liberação cirúrgica do ombro operado.

O outro $(6,7 \%)$ paciente que evoluiu com importante restrição das atividades diárias teve infecção aguda da ferida operatória, sendo tratado com múltiplos desbridamentos e antibiótico. Este paciente sofreu uma fratura multifragmentar do corpo da escápula (Ada e Miller tipo IV), tratada pelo acesso posterior "clássico" de Judet. Após vários procedimentos cirúrgicos de limpeza local, a infecção foi controlada, mas o paciente apresentou atrofia importante da musculatura infraespinal e, consequentemente, rotação externa não funcional. Apesar do protocolo agressivo de fisioterapia, ele mantém o déficit de rotação do ombro operado.

\section{ANÁLISE RADIOGRÁFICA}

$\mathrm{Na}$ última visita ambulatorial, todos os pacientes foram avaliados radiograficamente com radiografias simples, nas incidências em ântero-posterior, axilar e perfil da escápula. Um dos autores interpretou os exames. Não foram detectados sinais de perda de redução, pseudartrose ou OA pós-traumática (Figuras 3 e 4). Não houve impacto ou penetração articular pela presença do material de osteossíntese.

\section{DISCUSSÃO}

Atualmente, ainda não há consenso a respeito do tratamento das fraturas da escápula. Embora no passado tenham sido quase exclusivamente de manejo conservador, vem crescendo, nos dias de hoje, as indicações de abordagem cirúrgica destas lesões ${ }^{(14-17)}$. Isto se deve, principalmente, ao elevado número de resultados funcionais insatisfatórios observados após o tratamento conservador quando há a presença de desvio ou de instabilidade interfragmentar ou de rotura do CSSO. Anavian et al. observaram que, mesmo na ausência de desvio inicial, algumas fraturas extra-articulares podem desviar no curso do tratamento conservador ${ }^{(18)}$.

Em 1991, Ada e Miller relataram excelente resultado em oito pacientes tratados com fixação interna ${ }^{(3)}$. Mais recentemente, Schandelmaier et al. obtiveram aproximadamente $82 \%$ de resultados satisfatórios em 22 pacientes submetidos à fixação interna pela via "clássica" de Judet (16 casos) ou pela via anterior (seis) ${ }^{(19)}$. Dois anos mais tarde, Lantry et al. fizeram uma revisão sistemática das evidências sobre o tratamento cirúrgico das fraturas da escápula $^{(20)}$. Doze estudos foram incluídos (com um total de 163 casos), com uma média de seguimento de 53,3 meses. Embora haja uma fraqueza óbvia nos números finais, devido à falta de padronização nos sistemas de avaliação usados, foram observados $83,4 \%$ de excelentes e bons resultados nos trabalhos analisados por Lantry et $a l .{ }^{(20)}$. Em nosso estudo, resultados subjetivos e objetivos satisfatórios foram observados em 86,7\% (13 pacientes), com retorno completo às atividades sociais e profissionais. 


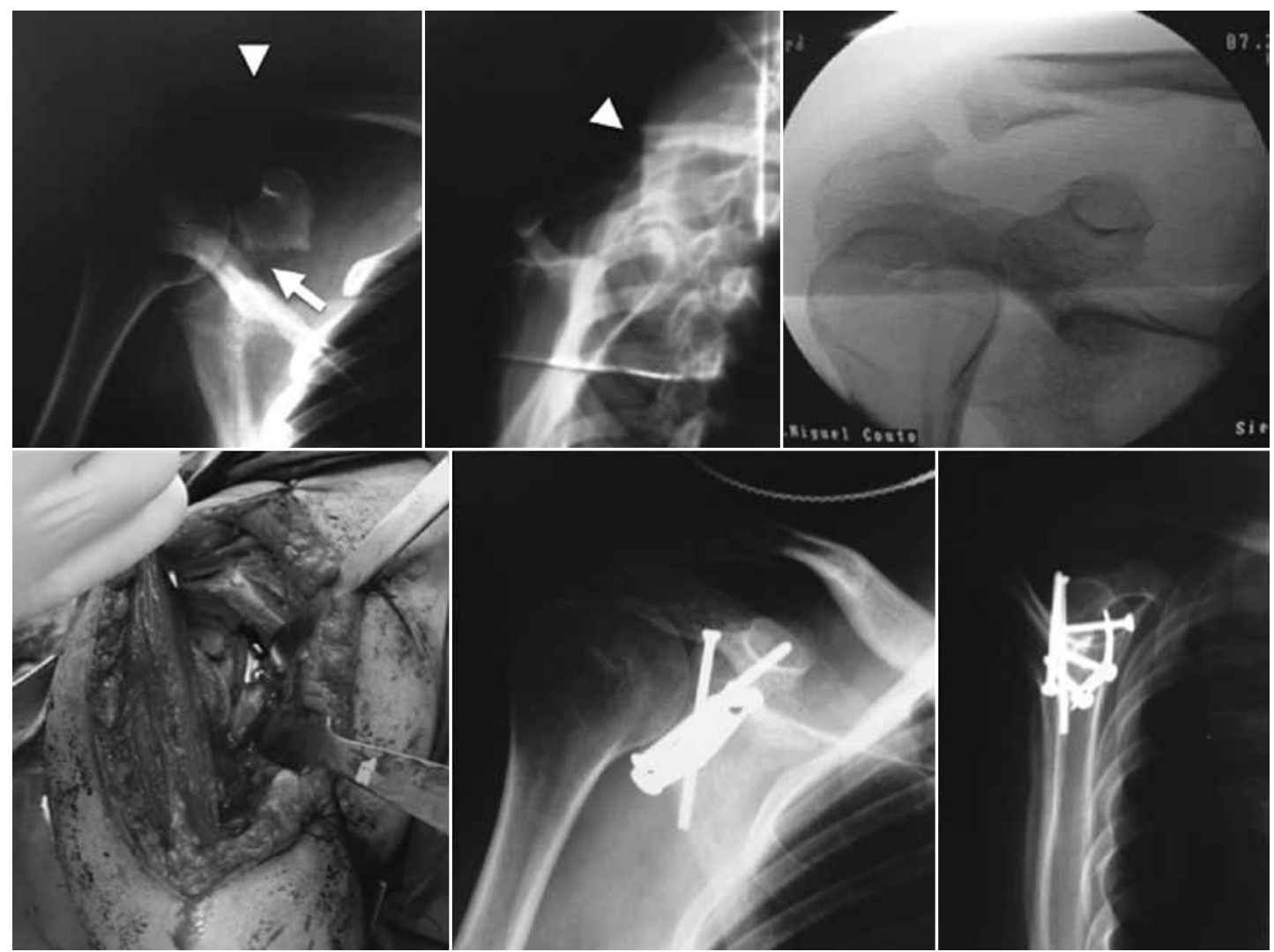

Figura 3 - Paciente do sexo feminino com rotura do complexo suspensório superior do ombro direito, caracterizada por fraturas do terço distal da clavícula e da cavidade glenoide (tipo III de Ada e Miller e 1B de Ideberg et al.). Notar as lesões mostradas pelas setas. Resultado final satisfatório, clínico e radiográfico.

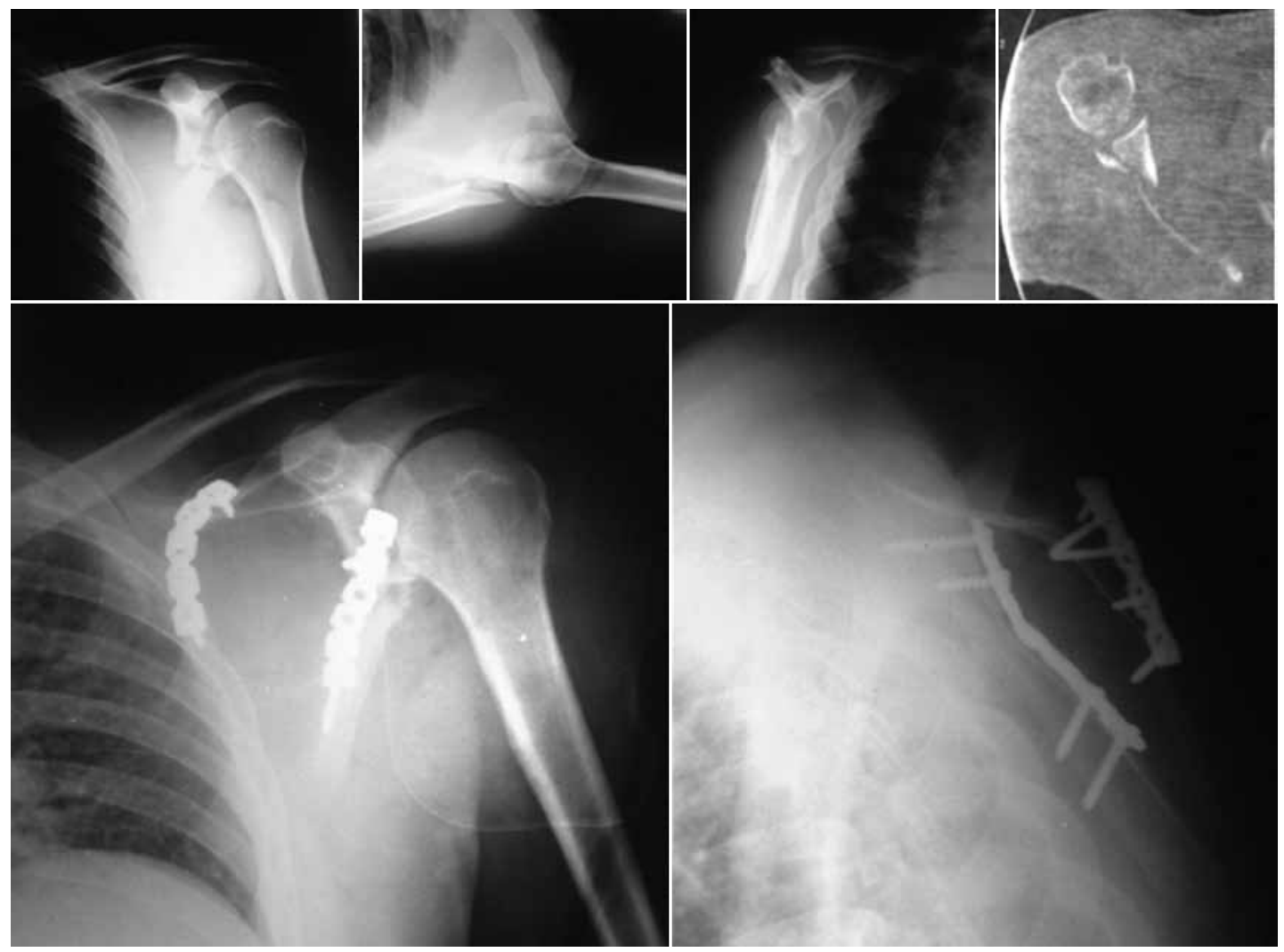

Figura 4 - Paciente do sexo masculino com fratura desviada da borda lateral da escápula, do tipo IIA de Ada e Miller. Abordado pela via posterior de Judet "modificada". Resultado final satisfatório, clínico e radiográfico. 
Uma das razões que creditamos aos excelentes e bons resultados vistos no atual estudo foi a escolha adequada da via de acesso. A abordagem posterior é a mais utilizada na literatura (preferencialmente a "clássica" de Judet), pois permite a exposição da superfície articular, do colo e do corpo da escápula ${ }^{(9)}$. A despeito de uma visualização adequada, alguns autores têm chamado a atenção para as maiores morbidade e complicações relacionadas à esta via, principalmente pela grande dissecação muscular das estruturas da cintura escapular $^{(10,21)}$. Em nossa casuística, utilizamos a via "clássica" em quatro dos 13 pacientes abordados por acesso posterior. Nos outros nove pacientes, utilizamos a modificação proposta por Obremskey e Lyman, com dissecação entre os músculos infraespinal e redondo menor. Conforme proposto pelos autores que modificaram a abordagem, é possível combinar a boa exposição das estruturas ósseas com os elementos neurovasculares maiores (nervo axilar e artéria circunflexa escapular). Outros autores descreveram modificações da via "clássica" de Judet na tentativa de minimizar a dissecação de partes moles e o risco de dano neurovascular ${ }^{(21-24)}$.

Acreditamos que o uso de abordagens mais limitadas possibilita melhores resultados pois permite o início mais precoce do protocolo de reabilitação e reduz a taxa de complicações. A exemplo do que se observa em outras lesões articulares e periarticulares complexas, a mobilidade precoce e livre no período pós-operatório devem ser encorajada ${ }^{(20,24)}$. No estudo de Landry et $a l$., as complicações mais comumente observadas foram infecção e capsulite adesiva, ambas com uma taxa de $4,2 \%^{(20)}$. A maioria dos casos de infecção foram resolvidos após antibioticoterapia, com ou sem drenagem cirúrgica. Em nosso estudo, observamos um caso de infecção superficial e outro de perda da mobilidade articular funcional. Coincidentemente, estes dois pacientes foram abordados pela via "clássica" de Judet. Ambos tiveram resultado final insatisfatório, a despeito do bom resultado radiográfico.

Em conclusão, a presente investigação demonstrou resultados preliminares satisfatórios em 13 dos 15 pacientes operados devido à fratura desviada ou instável da escápula. Diversos aspectos parecem ser relevantes na obtenção de resultados excelentes e bons, como a escolha da via de acesso e o protocolo de reabilitação. $O$ respeito aos tecidos moles é o fator principal no manejo destas lesões. Nossos achados corroboram a atual indicação de fixação interna em casos selecionados de fratura da escápula, principalmente quando há desvio ou instabilidade no corpo, no colo ou na cavidade glenoide.

\section{REFERÊNCIAS}

1. Cole PA. Scapular fractures. Orthop Clin North Am. 2002;33(1):1-18.

2. Weening B, Walton C, Cole PA, Alanezi K, Hanson BP, Bhandari M. Lower mortality in patients with scapular fractures. J Trauma. 2005;59(6):1477-81.

3. Ada JR, Miller ME. Scapular fractures. Analysis of 113 cases. Clin Orthop Rel Res. $1991 ;(269): 178-80$.

4. Bartoniček J, Cronier P. History of the treatment of scapula fractures. Arch Orthop Trauma Surg. 2009; published online on May 14 [Epub ahead of print].

5. Ideberg R, Grevsten S, Larsson S. Epidemiology of scapular fractures. Incidence and classification of 338 fractures. Acta Orthop Scand. 1995;66(5):395-7.

6. Advanced Trauma Life Support ${ }^{\mathrm{TM}}$ Student Manual. Chicago:American College of Surgeons, 2004

7. Goss TP. Fractures of the glenoid cavity. J Bone Joint Surg Am. 1992;74(2):299-305.

8. Goss TP. Scapular fractures and dislocations: diagnosis and treatment. J Am Acad Orthop Surg. 1995;3(1):22-33.

9. Judet R. Traitement chirurgical des fractures de l'omoplate. Acta Orthop Belg. 1964:30:673-8.

10. Obremskey WT, Lyman JR. A modified Judet approach to the scapula. J Orthop Trauma. 2004;18(10):696-9.

11. Hoppenfeld S. Surgical exposures in orthopaedics. Philadelphia:J.B. Lippincott Company, 1984.

12. Schofer MD, Sehrt AC, Timmesfeld N, Störmer S, Kortmann HR. Fractures of the scapula: long-term results after conservative treatment. Arch Orthop Trauma Surg. 2009;129(11):1511-9.

13. Constant $\mathrm{CR}$, Murley AH. A clinical method of functional assessment of the shoulder. Clin Orthop Relat Res. 1987;(214):160-4.
14. Veysi VT, Mittal R, Agarwal S, Dosani A, Giannoudis PV. Multiple trauma and scapula fractures: so what? J Trauma. 2003;55(6):1145-7.

15. Findlay RT. Fractures of the scapula. Ann Surg. 1931;93(5):1001-8.

16. Nordqvist A, Petersson C. Fractures of the body, neck, or spine of the scapula. Clin Orthop Rel Res. 1990;(283): 139-44.

17. Nau T, Petras N, Vécsei V. Fractures of the scapula. Classification and treatment principles. Osteo Trauma Care. 2004;12(4):174-9.

18. Anavian J, Khanna G, Plocher EK, Wijdicks CA, Cole PA. Progressive displacement of scapula fractures. J Trauma. 2010;69(1):156-61.

19. Schandelmaier P, Blauth M, Schneider C, Krettek C. Fractures of the glenoid treated by operation. A 5- to 23-year follow-up of 22 cases. J Bone Joint Surg Br. 2002;84(2):173-7.

20. Lantry JM, Roberts CS, Giannoudis PV. Operative treatment of scapular fractures: a systematic review. Injury. 2008;39(3):271-83.

21. Jones CB, Cornelius JP, Sietsema DL, Ringler JR, Endres TJ. Modified Judet approach and minifragment fixation of scapular body and glenoid neck fractures. $J$ Orthop Trauma. 2009;23(8):558-64.

22. Ebraheim NA, Mekhail AO, Padanilum TG, Yeasting RA. Anatomic considerations for a modified posterior approach to the scapula. Clin Orthop Rel Res. 1997;(334):136-43.

23. Kligman M, Roffman M. Posterior approach for glenoid fracture. J Trauma. 1997;42(4):733-5.

24. Nork SE, Barei DP, Gardner MJ, Schildhauer TA, Mayo KA, Benirschke SK. Surgical exposure and fixation of displaced type IV, V, and VI glenoid fractures. J Orthop Trauma. 2008;22(7):487-93. 